\title{
EXPERIMENTAL RESEARCH OF HIGHLY RELIABLE METHODS OF HYDROACOUSTIC COMMUNICATION FOR LIMITED WATER BODIES WITH LOW SOUND ABSORPTION AND A COMPLEX REVERBERATION PATTERN
}

\author{
Alexander Dikarev ${ }^{1}$, Stanislav Dmitriev ${ }^{1}$, Vitaliy Kubkin ${ }^{1}$, Arron Griffiths ${ }^{2}$ and \\ Arthur Abelentsev ${ }^{1}$ \\ ${ }^{1}$ Underwater communication and navigation laboratory, LLC, Moscow, Russia \\ ${ }^{2}$ The University of Manchester, Dalton Cumbrian Facility, Whitehaven, UK
}

\begin{abstract}
The article presents the results of a study of transmission reliability and the quality of determining the propagation time of multifrequency signals in conditions of confined water bodies which walls and bottom are made of materials with a low absorption coefficient of acoustic waves and with a complex reverberation pattern.
\end{abstract}

\section{KEYWORDS}

Hydroacoustic channel, channel impulse response, OFDM, multipath, reverberation, positioning system, confined water bodies

\section{INTRODUCTION}

The hydroacoustic channel typical for small, limited water bodies, such as technical tanks, settling ponds and various basins, has strong differences from the classical deep water and even from the shallow water channel. As noted in [1], the main differences are in the strictly limited amount of water, the geometry of the reservoir itself and the material of the walls and the bottom. Typically, the material of the walls, such as concrete/reinforced concrete or various metals, have an extremely low coefficient of absorption of acoustic waves. All these differences give an extremely complex impulse response of the channel mainly because the relaxation time is very significant, and the signal undergoes multiple reflections from the walls and the bottom. In this regard, the standard methods of hydroacoustic communication and especially methods for determining the propagation time of an acoustic signal are little or completely inapplicable. The relevance of the development of reliable positioning methods in small artificial reservoirs is noted, for example, in [2], [3] and in [4]. For brevity, we will refer to the collection of reservoirs and conditions described above as CLRE - Confined Long-Reverberating Environments.

\section{SIGNAL STRUCTURE}

During the experiments we used signal ensembles defined as follows:

$$
s_{m}=\frac{1}{N} \sum_{n=0}^{N-1} C_{m}(n) e^{j 2 \pi k / N}, m=1 . . M
$$

DOI : 10.5121/sipij.2018.9401 
Signal \& Image Processing : An International Journal (SIPIJ) Vol.9, No.4, August 2018

where $\mathrm{N}$ is the size of the discrete Fourier transform (FFT) window, $\mathrm{m}$ is the sequence number of the signal in the ensemble, and $\mathrm{M}$ is the ensemble size. $\mathrm{C}_{\mathrm{m}}(\mathrm{n})$ is some discrete function such that:

$$
C_{m}(n)=\left\{c_{m}\left(n-f_{c}\right), n=f_{c} . . f_{c}+N_{m} ; 0\right\}, n=0 . . N-1
$$

where $C_{m}(n)$ is the ensemble of pseudo-noise binary sequences (PNS), $m$ is the size of the ensemble, $N_{m}$ is the size of the sequence, $f_{c}$ is the shift of the argument of the function $C_{m}(n)$, such that $\mathrm{C}_{\mathrm{m}}(\mathrm{fc})=\mathrm{C}_{\mathrm{m}}(0)$.

As PNS ensembles $C_{m}$, M-sequences of length $4095\left(2^{12}-1\right)$ were used in the experiment and the $\mathrm{N}$ window size of the FFT was chosen equal to $2^{14}$ (16384). The ensembles of sm signals obtained according to (1) and (2) are frequency-modulated signals with orthogonal arrangement of subcarriers (so-called OFDM [5]), where the subcarriers are modulated according to the selected PNS, and the shift of argument $f_{c}$ determines the index (and, frequency) of the lower subcarrier. The use of signals of this type is aimed at solving the following problems: reducing the signal duration while simultaneously increasing the PNS length, maximizing the effective use of the frequency band, and increasing the noise immunity of the signal from frequency selective fading caused by multipath and reverberation [6].

\section{SCHEME AND DESCRIPTION OF THE EXPERIMENT}

The experiment was conducted in a metal tank size $3 \times 1.5 \times 1.2$ meters. The thickness of the material of the walls and the bottom of the tank is $5 \mathrm{~mm}$. In the tank, there are no means of acoustic muffling. The water temperature at the time of the experiments was $24.0{ }^{\circ} \mathrm{C}$. All measurements were made in a local coordinate system, the reference point of which is in the centre of the horizontal section of the tank at the water level, the vertical axis is directed downwards. In the experiments, 4 transmitters and a receiver were used. Their coordinates for all the experiments are summarized in Table 1.

Table 1. Coordinates of the receiver and transmitters in experiments

\begin{tabular}{|l|l|l|l|}
\hline Receiver/Transmitter & $\mathbf{X}, \mathbf{m}$ & $\mathbf{Y}, \mathbf{m}$ & $\mathbf{Z}, \mathbf{m}$ \\
\hline $\mathrm{T}_{1}$ & 0 & 0.7 & 0.5 \\
\hline $\mathrm{T}_{2}$ & 1.445 & 0 & 0.5 \\
\hline $\mathrm{T}_{3}$ & 0 & -0.7 & 0.5 \\
\hline $\mathrm{T}_{4}$ & -1.445 & 0 & 0.5 \\
\hline $\mathrm{R}_{1}$ & -1.125 & 0 & 0.5 \\
\hline $\mathrm{R}_{2}$ & 0 & 0 & 0.5 \\
\hline $\mathrm{R}_{3}$ & 1.125 & 0 & 0.5 \\
\hline
\end{tabular}

Separate experiments consisted of recording signals at the points $R_{1}, R_{2}$ and $R_{3}$, radiated cyclically with the help of transmitters $T_{1}, T_{2}, T_{3}, T_{4}$. At each of the points $R_{1 \cdot .3}, 5$ records were obtained, every of which contains 250 signals from each of the transmitters $\mathrm{T}_{1 . .4}$, which gives a total of 1250 signals for particular receiver position (5000 signals in the sum for four transmitters). In this case, each transmitter has its own PNS and, therefore, its signal from the ensemble $S_{\mathrm{m}}$. As part of the experiment, the delay td between the time of the beginning of the emission of signals from each of the transmitters was 500 milliseconds. The sampling frequency $F_{\mathrm{s}}$ in all the experiments is $96 \mathrm{kHz}$, the size of the FFT conversion window is $\mathrm{N}=16384$, the frequency shift is $\mathrm{f}_{\mathrm{c}}=2048$. Thus, the width of the used frequency band $\mathrm{B}$ is:

$$
B=\frac{F_{s}}{N} N_{m} \approx 23994.14 H z
$$


In this case, the lower frequency of band B is:

$$
f_{l}=\frac{F_{s}}{N} f_{c}=12000 H z
$$

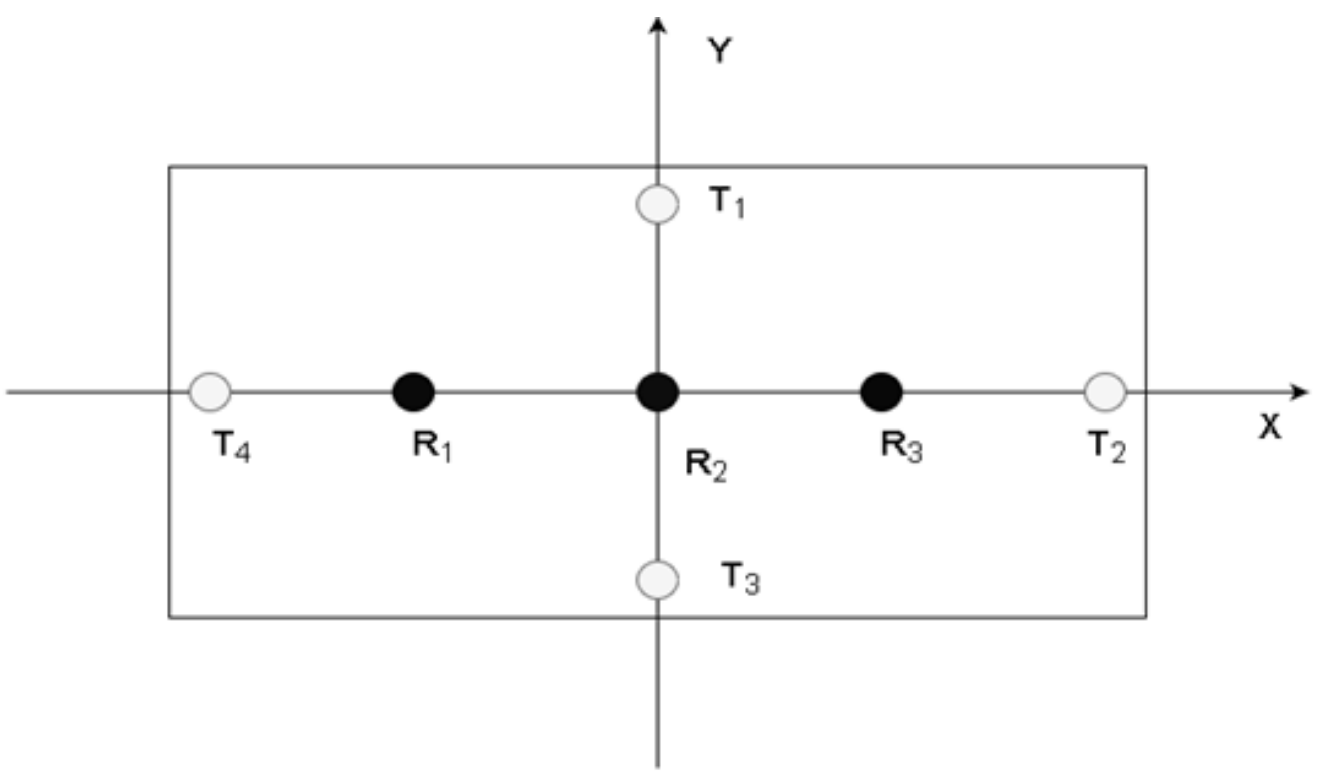

Figure 1. Scheme of the experiment

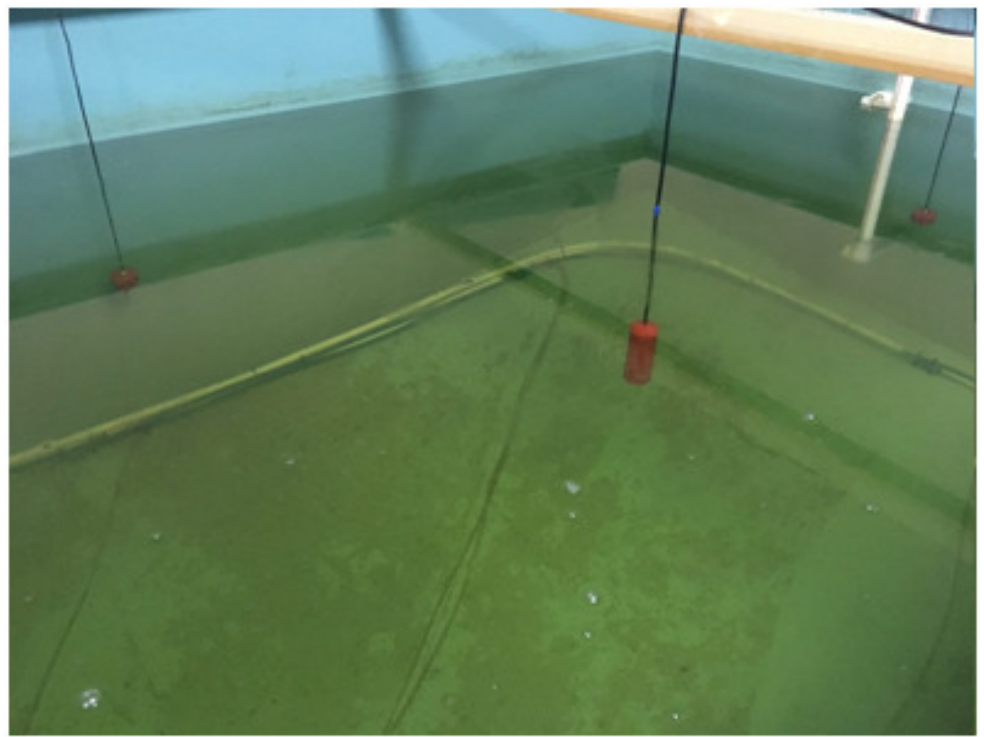

Figure 2. Experimental equipment

Figure 2 shows two of the four transmitters (along with the sides of the pool) and the receiver (in the middle). The timing of the arrival of signals from the transmitters $T_{1} . . T_{4}$ is determined by means of matched filtering. The response of the matched filter is determined by correlation with the template $T_{m}$ obtained because of the $m$-th sequence from ensemble $C_{m}$ : 
Signal \& Image Processing : An International Journal (SIPIJ) Vol.9, No.4, August 2018

$$
p(t)=\mid \operatorname{Re}\left(s(f) \cdot \operatorname{conj}\left(T_{m}(f)\right) \mid\right.
$$

Where $\mathrm{S}(\mathrm{f})$ is the spectrum of the input signal in the processing window. The signal-to-noise ratio at the output of the matched filter is determined from (6):

$$
S N R=P_{\max }^{2} \cdot N / \sum_{t=0}^{N} P(t)-P_{\max }^{2}(t)
$$

Figures 3 and 4 show the receiver and transmitter circuits, respectively.

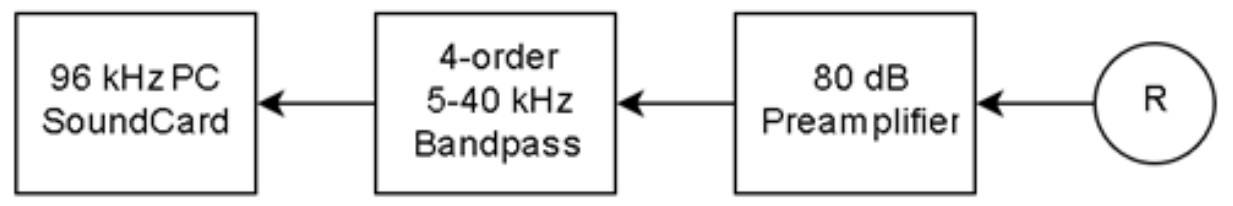

Figure 3. Receiver circuit

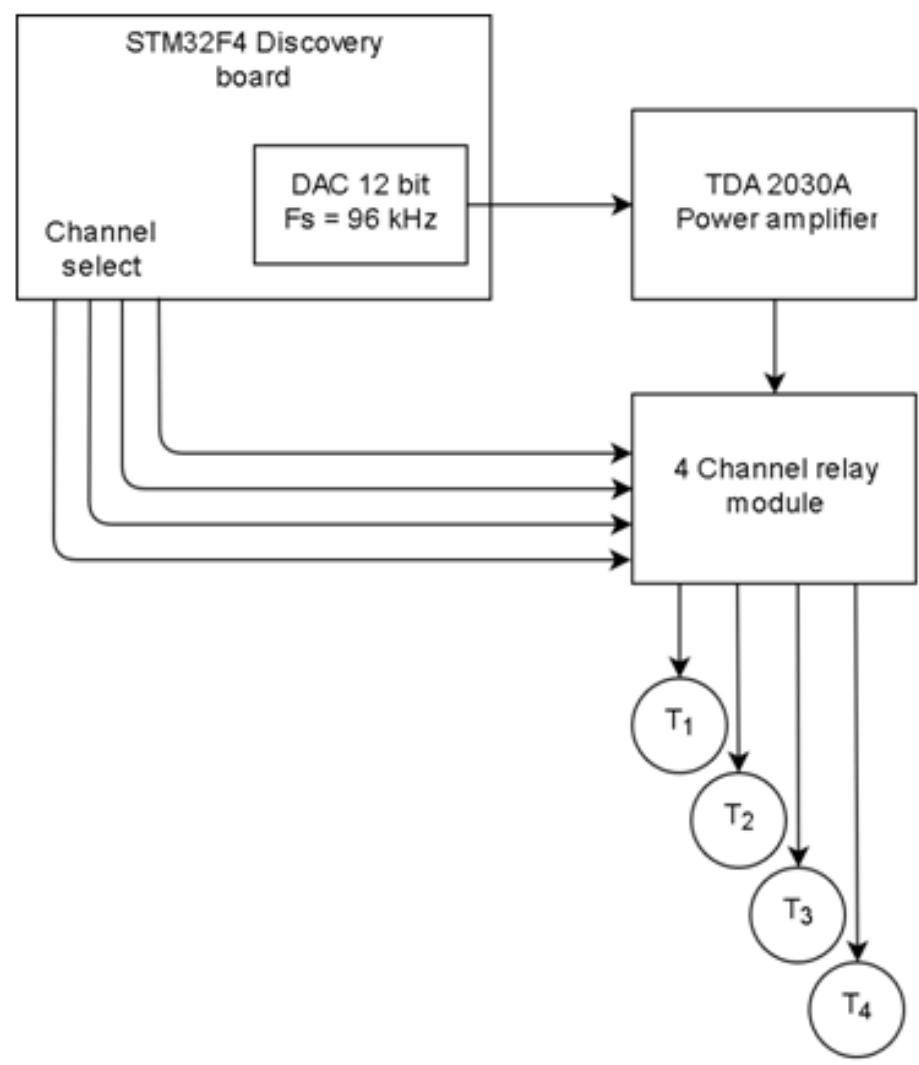

Figure 4. Transmitter circuit

As a signal generator, the STM32F4 Discovery board is used. The signal from the DAC board is sent to the power amplifier, and with the help of the 4-channel relay unit, the amplified signal is fed to the corresponding transmit antenna. 
According to the arrangement of the transmitting antennas and subtracting the fixed delay between the radiation from different channels, and assuming the sound speed for the given conditions $\left(\mathrm{t}=24{ }^{\circ} \mathrm{C}, \mathrm{s}=0\right.$ PSU, $\left.\mathrm{p}=0.04903 \mathrm{bar}\right)$ according to [7] equal to $1493.9 \mathrm{~m} / \mathrm{s}$, the difference in the arrival times of the signals should correspond to the data given in Table 2:

Table 2. Estimated differences in arrival times

\begin{tabular}{|l|l|l|l|}
\hline Receiver & $\mathbf{T}_{\mathbf{1}}-\mathbf{T}_{\mathbf{2}}, \mathbf{m s e c}$ & $\mathbf{T}_{\mathbf{1}}-\mathbf{T}_{\mathbf{3}}, \mathbf{m s e c}$ & $\mathbf{T}_{\mathbf{1}}-\mathbf{T}_{\mathbf{4}}, \mathbf{m s e c}$ \\
\hline $\mathrm{R}_{1}$ & -0.834 & 0 & 0.673 \\
\hline $\mathrm{R}_{2}$ & -0.499 & 0 & -0.499 \\
\hline $\mathrm{R}_{3}$ & 0.673 & 0 & -0.834 \\
\hline
\end{tabular}

In this case, according to the sampling frequency used, $F_{s}=96 \mathrm{kHz}$, the time resolution is $1 / 96=$ $0.010417 \mathrm{msec}$. It is assumed that during one radiation cycle for all four channels the sampling rate is stable.

\section{EXPERIMENTAL RESULTS}

Figure 5 shows the typical response of a matched filter (impulse response of a channel). The impulse response illustrates the complex nature of the reverberation pattern. The allocation of the "direct ray" is difficult - the position of the maximum does not correspond to the actual beginning of the signal (see Figure 6) and is related to the summation of the re-reflected signal. This situation is typical for CLRE. It is worth mentioning that in addition to the described signals with base 4095, attempts were made to use signals with a smaller base (2047 and 1023), but, in view of their worse correlation properties, they do not provide $100 \%$ detection under the conditions described, unlike signals with base 4095 .

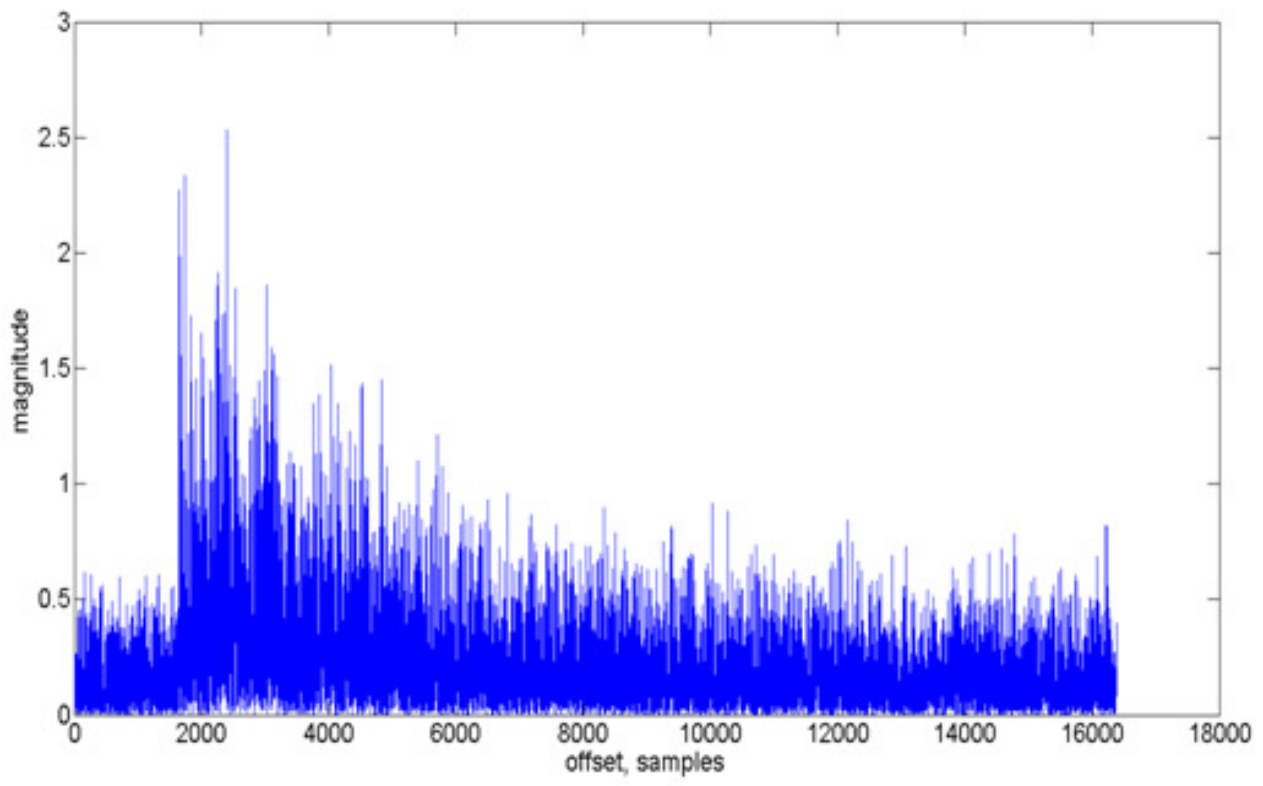

Figure 5. Typical impulse response in one of the experiments

In Figure 6, the response of the matched filter is superimposed on the time domain of the received signal, from which it becomes clear that the actual position of the signal beginning cannot be reliably estimated by the maximum response of the matched filter. Instead, to determine the true arrival time of the "direct" ray was used as an empirical rule: the direct "ray" is the extremum of 
the response of the matched filter, which is earlier than the global maximum, no more than 2000 samples, with a value of at least $4.1 \mu$, where $\mu$ is the average value in the response of the matched filter. This approach allowed to obtain the experimental values of the times of arrival times closest to the calculated ones, the values obtained are summarized in Table 3 . The value of 2000 samples is determined mainly by the difference in the path of the direct beam and the sum of the reflected signals, which gives the maximum in the response of the matched filter. This value was obtained by direct analysis of the total volume of the received impulse response of the channel (responses of the matched filter). Factor 4.1 was chosen experimentally, starting from the conditions for minimizing the discrepancies between the calculated and experimental data.

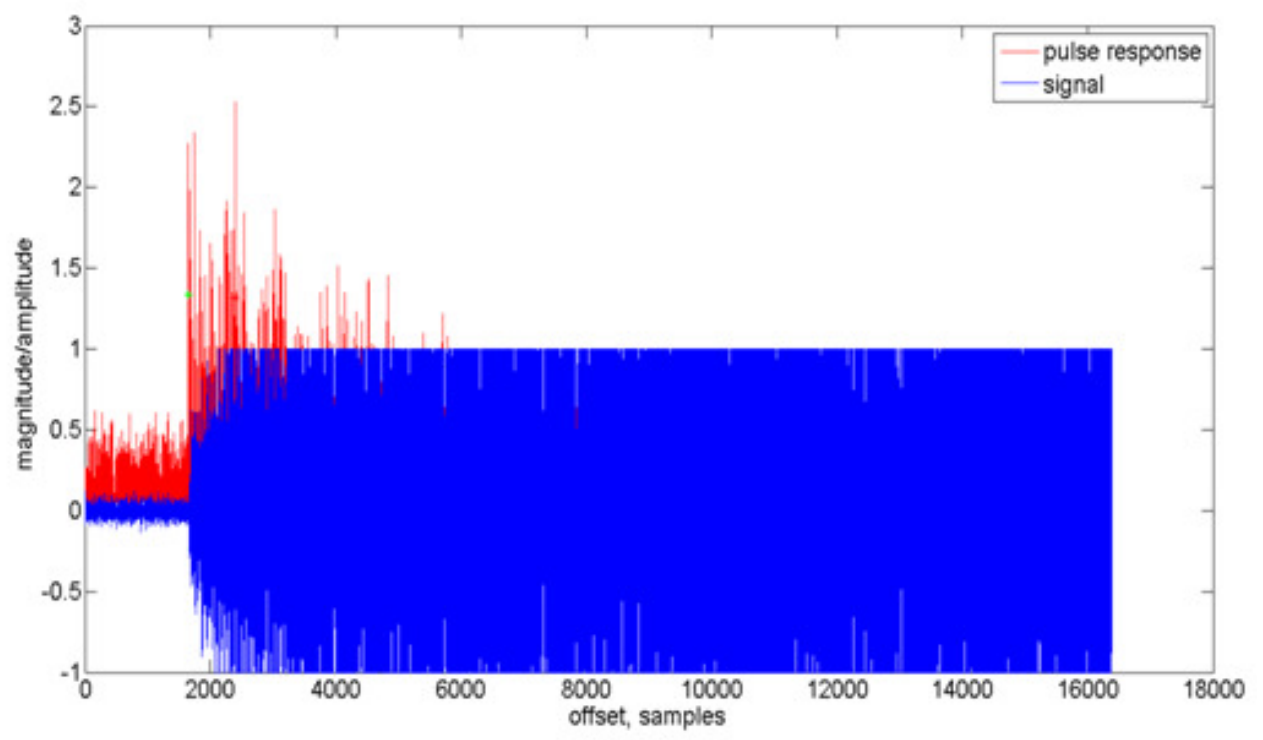

Figure 6. Received signal in the time domain with the superimposed response of the matched filter

Table 3. Experimental differences in arrival times with the corresponding values of root-mean-square deviation

\begin{tabular}{|l|l|l|l|}
\hline Receiver & $\mathbf{T}_{\mathbf{1}}-\mathbf{T}_{\mathbf{2}}(\mathbf{s t d}), \mathbf{m s e c}$ & $\mathbf{T}_{\mathbf{1}}-\mathbf{T}_{\mathbf{3}}$ (std), $\mathbf{m s e c}$ & $\mathbf{T}_{\mathbf{1}}-\mathbf{T}_{\mathbf{4}}(\mathbf{s t d}), \mathbf{m s e c}$ \\
\hline $\mathrm{R}_{1}$ & $-1.101(0.042)$ & $0.036(0.011)$ & $0.687(0.015)$ \\
\hline $\mathrm{R}_{2}$ & $-0.495(0.012)$ & $0.007(0.012)$ & $-0.520(0.015)$ \\
\hline $\mathrm{R}_{3}$ & $0.695(0.015)$ & $-0.014(0.012)$ & $-1.167(0.031)$ \\
\hline
\end{tabular}

Corresponding obtained values of standard deviation (see Table 3) allow considering the obtained results as reliable. The discrepancy with the calculated differences expressed in distance measures is given in Table 4.

Table 4. Discrepancies between experimental data and estimated (pseudo-ranges)

\begin{tabular}{|l|l|l|l|}
\hline Receiver & $\mathbf{E} \mathbf{d}_{\mathbf{1}}-\mathbf{d}_{\mathbf{2}}, \mathbf{m}$ & $\mathbf{E} \mathbf{d}_{\mathbf{1}}-\mathbf{d}_{\mathbf{3}}$, & $\mathbf{E} \mathbf{d}_{\mathbf{1}}-\mathbf{d}_{\mathbf{4}}$, \\
\hline $\mathrm{R}_{1}$ & 0.399 & -0.054 & -0.021 \\
\hline $\mathrm{R}_{2}$ & -0.006 & -0.011 & 0.031 \\
\hline $\mathrm{R}_{3}$ & -0.033 & 0.021 & 0.497 \\
\hline
\end{tabular}

The values in Table 4 are substantially smaller than the dimensions of the transducers. One value is the order of the dimensions of the converter $(-0.054 \mathrm{~m})$, and the two values reach a value of the order of $40 \mathrm{~cm}$ (shown in bold). Characteristically, the values with the greatest error correspond to those reciprocal arrangements of the transmitters and the receiver, at which the maximum distance between the receiver and one of the transmitters is reached (experiments $R_{1}$ and $R_{3}$, 
transmitters $T_{2}$ and $T_{4}$, respectively). Remarkably, the results obtained in the experiment $R_{1}$ with the data obtained in the $\mathrm{R}_{3}$ experiment are in good adjustment (both mutual arrangements are geometrically mirror-like).

Figure 7 shows a graph of the difference in the arrival times between the transmitters $T_{1}$ and $T_{4}$ in the $R_{3}$ experiment on a sample of 365 values.

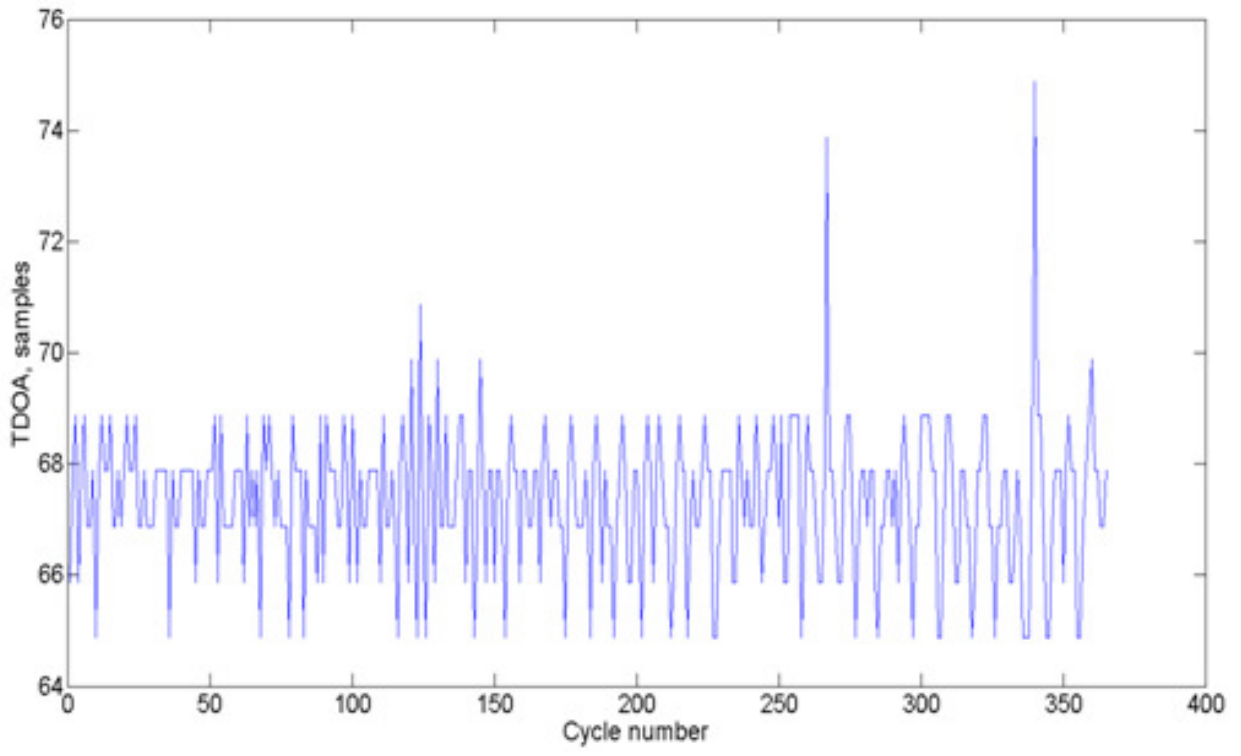

Figure 7. TDOA values for transmitters $T_{1}$ and $T_{4}$ in experiment $R_{3}$

The graph in Fig. 7. Also allows to talk about the reliability of the results - the range of values varies within 5-6 samples. Figures 8-10 show histograms of the distribution regarding delays between the direct and maximum ray. Based on this data, it can be argued that the delay between the direct and the maximum ray takes values from a limited range. This situation can be explained by the presence of stable maxima of the interference pattern, the relative magnitude of which varies: the global maximum "jumps" between a fixed set of stable positions. The very fact of the presence of the maximum ray is explained by the fact that the total energy of the reflections can significantly exceed the energy of the direct ray. This is the main difference CLRE from the conditions of natural water bodies, where a significant part of the energy is dissipated.

To explain the shape of the distributions shown in Fig. 8-10, additional research is required. 
Signal \& Image Processing : An International Journal (SIPIJ) Vol.9, No.4, August 2018
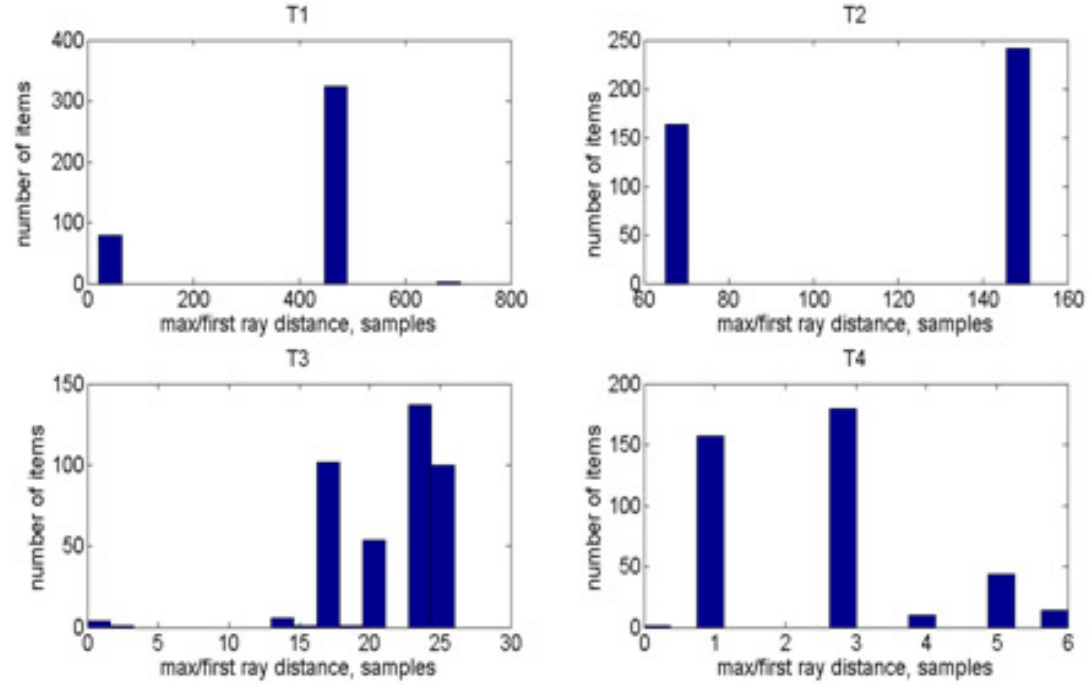

Figure 8. Distribution of delays between direct and maximum rays (Experiment $\mathrm{R}_{1}$ )
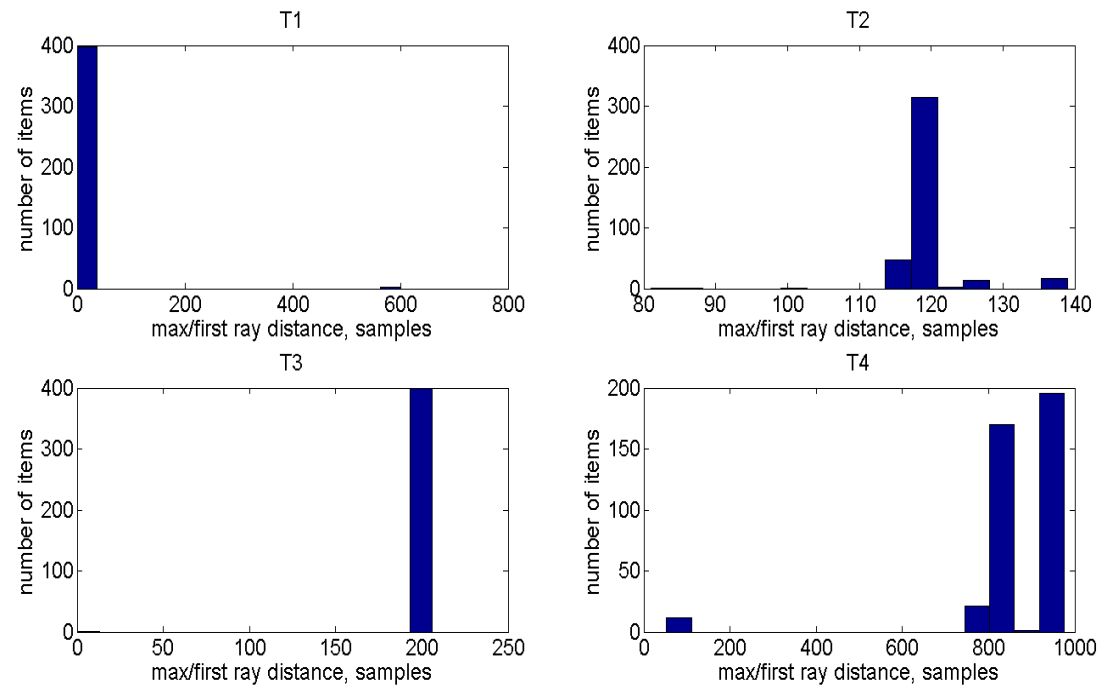

Figure 9. Distribution of delays between direct and maximum rays (Experiment $\mathrm{R}_{2}$ ) 

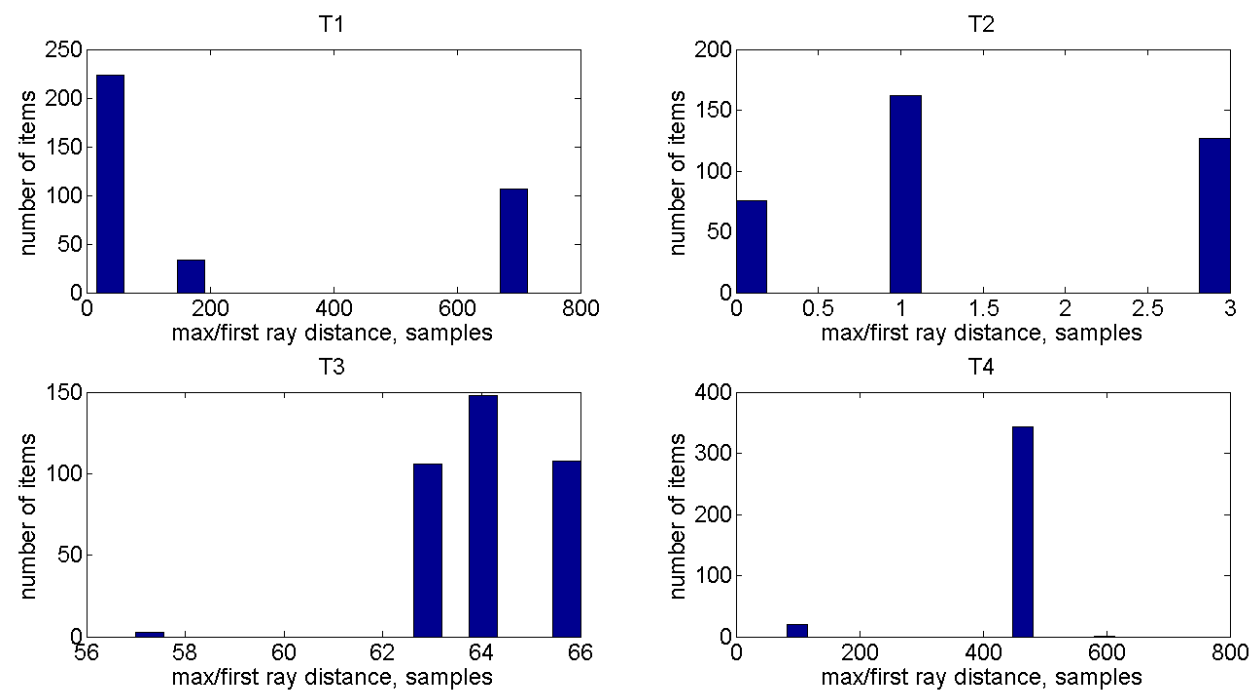

Figure 10. Distribution of delays between direct and maximum rays (Experiment $\mathrm{R}_{3}$ )

\section{CONCLUSIONS}

According to the results of the study, one can state:

- CLRE specific hydroacoustic channel is characterized by a very complicated impulse response, wherein the overwhelming majority of cases the position of the peak of the impulse response determines only the position of one of the maxima of the interference pattern and does not determine the position of the direct ray which amplitude is much smaller;

- a stable determination of a useful signal presence can only be ensured if a length of a PN sequence is at least 4095 is used;

- The most effective modulation method is OFDM, which provides better spectral efficiency (compared to PSK, FSK);

- The developed empirical rule for estimating the position of the direct ray makes it possible to determine the time differences of arrival (for navigating) in most cases with an accuracy of the order of several samples (comparable to the size of the hydroacoustic antennas used), with an upper limit of 30 samples;

- in CLRE conditions it is possible to build a navigation system using equipment that utilizes the sampling frequency of $96 \mathrm{kHz}$;

The objectives of the further research are:

- analysis of the distributions of the path differences between the direct and maximum ray;

- the construction of a navigation system based on the described signal and methods of its processing; 


\section{ACKNOWLEDGEMENTS}

The work is supported by:

- the Skolkovo Foundation www.sk.ru;

- the Foundation for Assistance to Small Innovative Enterprises in the Scientific and Technical

Sphere www.fasie.ru;

\section{REFERENCES}

[1] Dikarev, A., Griffiths, A., Watson, S., Lennox, B., Green, P.R., Combined multiuser acoustic communication and localization system for uAUVs operating in confined underwater environments, 2015. IFAC Workshop on Navigation, Guidance and Control. Girona, Spain

[2] Hussain, M., Trigoni, N., Distributed localization in Cluttered Underwater Evironments, The Fifth ACM International Workshop on Underwater Networks (WUWNet), Sept-Oct 2010, Woods Hole, Massachusetts, USA.

[3] Nawas, M., Hussain, S., Watson, S., Trigoni, N., Green, P.N., An underwater Robotic Network for Monitoring Nuclear Waste Storage Ponds, In Sensor Systems and Software, Springer 2009. (ISSN 1867-8211 ISBN-10 30642-11527-6, ISBN-13987-3-642-11527-1).

[4] York, T.A., Green, P.N., Green, P.R., Phasouliotis, A., Qu, Z., Watson, S., Hussain, M., Nawaz, S., Trigoni, N., Stanley, S., "Acoustic Sensor Networks for Decommisioning", Measurement and Control, Vol.45/2, pp.48-54, March 2012.

[5] Yang, J. Ching-Nung (October 10, 2001), "What is OFDM and COFDM?". Shoufeng, Hualien 974, Taiwan: Department of Computer Science and Information Engineering National Dong Hwa University. Retrieved 2017-04-16.

[6] Ahmed Alshammari, Saleh Albdran. Effects of Fading Channels of OFDM, IOSR Journal on Engineering (IOSRJEN), Volume 2, Issue 9 (September 2012), pp. 116-121

[7] G.S.K. Wong ans S Zhu, Speed of Sound in seawater as a function of salinity, temperature and pressure (1995) J. Acous. Soc. Am. 97(3) pp. 1732-1736

\section{AUTHORS}

\section{Alexander Dikarev}

received his M.Eng in Launching equipment of rockets and cosmic apparatus from Volgograd Technical State University, Russia. He has 10 years experience in underwater acoustic communication and navigation system design and development: in Research Insitute of Hydroacoustic Communications (Volgograd, Russia), The University Of Manchester (UK), now he is R\&D Director in Underwater communication \& Navigation laboratory (Moscow, Russia)

\section{Stanislav Dmitriev}

received his M.Sc in Radiophysics in Volgograd State University, Russia. He has 10 years of experience in Underwater Acoustic communication \& navigation system design \& development: in Research Insitute of Hydroacoustic Communications (Volgograd, Russia), now he is Engineering Director in Underwater Communication \& Navigation laboratory (Moscow, Russia)

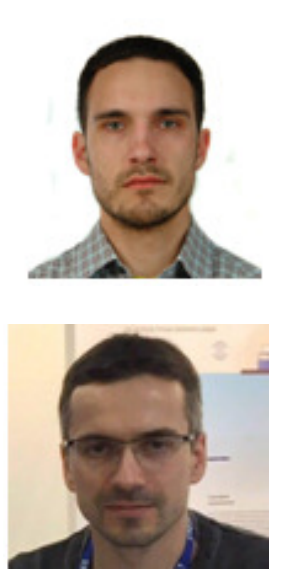


Signal \& Image Processing : An International Journal (SIPIJ) Vol.9, No.4, August 2018

\section{Vitaly Kubkin}

received his M.Eng in Volgograd Technical State University, Russia. He has 10 years of experience in Underwater Acoustic communication \& navigation system design \& development: in Research Insitute of Hydroacoustic Communications (Volgograd, Russia), now he is Senior Researcher in Underwater Communication \& Navigation laboratory (Moscow, Russia)

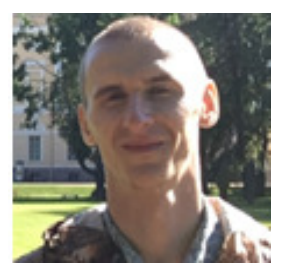

\section{Arron Griffiths}

Received his MSc in Plymouth University, UK. He has 10 years of experience in electronics and robotics, including underwater robotics. He is robotics engineer and assosiate researcher in The University Of Manchester, developing the AVEXIS-Mini vehicle. This is a micro ROV, designed to explore the legacy pond facilities on the Sellafield site.

\section{Artur Abelentsev}

received his M.Ec in Management in Kuban State Technological University, Russia. He has more than 15 years of experience in IT, embedded system architect and research management. $\mathrm{He}$ is a CEO in Underwater Communication \& Navigation laboratory (Moscow, Russia).

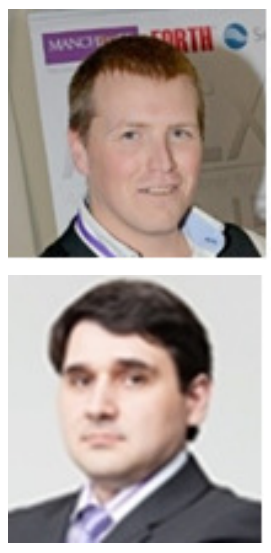

